\title{
COVID-19 and plastic surgery: a UK plastic surgery unit experience
}

\author{
Haitham Khashaba ${ }^{1}$ (D) Lisa Ng $^{1} \cdot$ Omer Osmani $^{1} \cdot$ Richard Chalmers ${ }^{1}$
}

Received: 21 May 2020 / Accepted: 2 July 2020 / Published online: 20 July 2020

(C) Springer-Verlag GmbH Germany, part of Springer Nature 2020

The COVID-19 pandemic has caused a devastating impact on health and economy across the whole world [1]. As a consequence of emergency planning and the shift of service provision, plastic surgery departments across the United Kingdom (UK) have had to quickly adopt new techniques and change existing practice in order to maintain and deliver essential plastic surgery services $[2,3]$.

The Plastic Surgery Department at the University Hospital of North Durham serves a population of more than 1 million [4] across the northeast region of the United Kingdom. One of the main challenges during the pandemic has been to safeguard the standards of care for patients and staff whilst delivering an effective and efficient service within a hospital infrastructure that has changed beyond all recognition.

We would like to share our experience in managing the skin cancers and plastic surgery trauma at our hospital. The aim is to highlight the modifications in the services to suite the requirements during the COVID-19 pandemic and identify areas of good practice that we aim to carry through to our practice as the service begins to normalise.

In the UK, as with many other European countries, theatres have been converted to ventilation pods to cope with the influx of COVID-19 patients and anaesthetic support staff has been re-deployed to cope with the influx of patients through the emergency department. This invariably resulted in severe

Haitham Khashaba

Haitham.khashaba@nhs.net

Lisa $\mathrm{Ng}$

lisa.ng@doctors.org.uk

Omer Osmani

omer.osmani@nhs.net

Richard Chalmers

richard.chalmers@nhs.net

1 Plastic Surgery Department, University Hospital of North Durham, Durham, UK reduction in the hospital capacity to perform elective procedures, which have basically been put on hold. National guidelines were published quickly on how to triage cancer patients according to clinical priority as well as treatment guidelines on management of specific cancer sites including skin cancer [5, 6]. Regular meetings with the cancer teams within the hospital as well as the Specialist Skin MDT managed to work through all the numerous guidelines and changing protocols, to come up with a system that could work for our population and service setup.

Alongside a new urgent daily cancer review clinic, we implemented a see and treat service with our dermatology colleagues to reduce additional hospital visits. All patients had the same safeguards in place that existed prior to the pandemic including a full clinical history, examination, clinical photography and detailed consent procedure to ensure that they were suitable for this setting. If suitable, they underwent surgery on the same day under local anaesthetic in the 2 dermatology theatre suites in the department. If not, then other options such as using the one main theatre list in the hospital was considered or more likely deferring surgery until a later date.

Throughout this period of the pandemic, we have continued to offer management of suspected melanoma and non-melanoma skin cancer (NMSC) in collaboration with the dermatology department. Before the pandemic, the two departments ran alongside each other, but not in conjunction with each other. The diagnostic clinical examination was done by the dermatologists, whilst the surgical management was covered by the plastic surgeons. This setup of "see and treat" clinic included screening of patients with skin lesions referred by the general practitioners as two-week wait referrals (as per British Association of Dermatologist guidelines) [7]. The screening process also involved use of referral letters aided by telemedicine. The objective of this setup was to provide an efficient service to the patients and also reduce the number of visits for the patient to the 
hospital, which was essential to reduce the actual footfall in the hospital as a measure to contain the spread of COVID-19 virus.

Our dermatology department has the advantage of having two treatment rooms which, during normal periods, are used for small skin procedures and simple biopsies. We made plans and provisions to use these treatment rooms as our main operating areas during the pandemic for skin cancer as well as minor trauma. It involved mobilising outpatient staff and resources to cover these lists. As part of the planning, we made sure the treatment rooms were deemed "safe" for the patients and staff in view of the appropriate (personal protective equipment) PPE requirements and social distancing in the waiting room. We also defined areas for donning and doffing to maintain an acceptable level of safety before, during and after the procedures. There was a small learning curve in getting used to the new operating environment, especially with the evolving advice surrounding PPE. We mention this as some colleagues found it difficult to use the surgical loupes with the FFP3 masks and visors and maintain a protective seal. The additional advantage of the treatment rooms was the access from the clinics, as they are all lined up in the same corridor.

During a period of 6 weeks from the 23rd of March, we performed 344 local anaesthetic procedures. On auditing our rate of complete excisions and complications in nonmelanoma skin cancer (NMSC), we have found that our rate of complete excision was $95.8 \%$ compared with $96.7 \%$ in a previous audit performed on a similar cohort of patients, prior to the pandemic. The difference was not deemed statistically significant using the chi-square test. We feel, anecdotally, one of the reasons for the slight fall in excision rates was the learning curve mentioned above in operating under new and challenging conditions. On the other hand, the specificity has increased to $99 \%$ and our likelihood to correctly exclude NMSC (negative predictive value) has significantly increased from 69 to $83 \%$.

A success story from this period is that all cancer cases were being seen and managed inside the national cancer targets and by the second week of lockdown, both the dermatology and the plastic surgery waiting lists for urgent cases including wide local excisions for pTis and pTla cases were cleared. This also allowed us to move into operating upon the cases prioritised as soon-normally operated on by 6 weeks, as well as those BCC cases in high risk areas as well as some of the other more routine BCC's on the waiting list. This rational was with a mind on starting to manage the back$\log$ of non-urgent that are building up and will be a major issue for NHS services after the pandemic resolves. Recent data has shown that whilst at the beginning of the pandemic the influx of new referrals had reduced, 8 weeks on from the start of the reconfiguration, our referrals are back to normal levelsapproximately 120 urgent cases per week with $50 \%$ of those being seen requiring a diagnostic biopsy or excision. Focusing on how to stay on top of the changing demands of the skin cancer service has been one of the many challenges throughout this period.

All patients who came to the department are provisionally screened for symptoms of COVID-19 by use of questionnaire and recording of temperature at the time of their visit. In addition, we are also swab testing patient for COVID now that the test is more readily available. Post-operative instructions were given to patients and those who required a second review were given an appointment or access details to the plastic surgery dressing clinic, run separately, for wound related care. Almost all patients had dissolvable sutures used to reduce the need for a dressing clinic appointment. Histology results were given to the patients by dedicated skin cancer nurse specialist over the phone. Patients requiring further treatment were given the option and rebooked for appropriate procedures.

We also ensured that the quality of our specialist skin multidisciplinary team meetings was maintained throughout this period whilst supporting local distancing recommendations. The team remained quorate throughout this period using remote access technology. Using in particular the revised UK Melanoma Focus guidelines, melanoma patients (pT2a and above with additional $\mathrm{pT} 1 \mathrm{~b}$ with high risk features) requiring wide local excision with the option of sentinel node biopsy (SLNB) were offered WLE with $1 \mathrm{~cm}$ margins and a delayed SLNB at a later unknown date. All these patients had their WLE in under 2 weeks of diagnosis and the SLNB service has now resumed after a 6-week delay starting with the fewer complex cases being performed at another hospital site within our NHS Trust deemed a "clean" site than the main hospital.

The role of the cancer nurse specialists during this time has also been invaluable with the development of a rapid access clinic, telephone diagnosis, and follow-up clinics as well as a dedicated secure email service to allow patients to email pictures of concerning lesions. Development of a combined skin cancer clinic was planned before the pandemic and many of these practices adapted during COVID-19 will be used to drive forward the planning and delivery of a more efficient and effective service for our cancer patients.

In order to maintain segregation of COVID-related admissions from non-COVID emergencies, our plastic surgery team helped to organise a multi-specialty area (MSA) in the Emergency Department (ED). This area is staffed by consultants and registrars from various surgical and medical specialities. This has been designed to off-load the ED from the patients they would usually see, who are channelled into the MSA department, to then concentrate their expertise on managing the high numbers of suspected COVID patients who are 
moved into a separate COVID part of the ED. The two areas were physically divided to facilitate safe patient flow through the hospital.

The normal setup for plastic surgery trauma is that the majority of patients get referred into and seen in the plastic dressing clinic (PDC). In the initial few weeks of the pandemic, the department ran with skeleton staff with the majority of patients being seen downstairs in the front of house MSA. As the structure of the hospital evolved, our PDC was needed to house COVIDnegative patients. Without a department to see all our patients including post-operation and those with complex wounds, we were then able to identify a larger department - vacated by ophthalmologist who were also being relocated where we moved our dressing clinic to. Patients with minor trauma were managed in the MSA and discharged from the ED. Those requiring a more formal surgical procedure or exploration were treated either in the emergency theatres (for general anaesthetics) or the plastic surgery dressing clinic treatment rooms (under local anaesthetics). We performed 190 local anaesthetic procedures and 42 emergency general anaesthetic procedures during this period. Despite the UK lockdown which restricted movement and recreation to only essential trips only, our trauma workload remained almost the same compared with a similar period last year. In the initial 6 weeks of the lockdown we have seen 468 patients ( 381 adult patients and 87 children) in the trauma setting, compared with 463 patients in a similar period in 2019. A possible reason is that despite the numbers of cases being referred to us from the ED across the county in particular for hand fractures had reduced, the number of cases usually seen and managed by the ED team that we were now managing had increased.

A technique that has shown its usefulness during the pandemic is Wide Awake Local Anaesthetic No Tourniquet (WALANT) as popularised by Dr. Lalonde [8]. The British Society for Surgery of the Hand (BSSH) has actively encouraged the use of WALANT and has produced guidance for its application [9]. In a selected group of patients, we have found this to be beneficial in facilitating local anaesthetic hand surgery procedures during the pandemic with limited access to main theatres that we would otherwise perform under general anaesthetic or regional block. The technique has been successfully used in flexor and extensor tendon repairs and exploration of wounds in both the plastic surgery dressing clinic and the dermatology theatre suite. All procedures done with this technique are currently under review and early results on follow-up are promising.

In conclusion, we would like to mention that throughout the world, this pandemic has changed the way people live and work. Our experience during this time has been, as many would put it, "a once in a lifetime global event". It has made us think about the ways we can offer high standard of care for our patients within difficult and changing situations with limited resources in often different clinical settings to those that we are used to. The major safety concerns surrounding patients and staff from COVID-19 have been ever present in all we do, but at the centre of it all is the drive to keep treating patients whilst maintaining the high standards we expect from the NHS. As plastic surgeons, we train ourselves based on Sir Harold Gillies Ten Commandments, one of which says "thou shalt not have a routine" [10]. The ability to adapt and evolve has meant that new skills have been learnt and good practice working alongside other colleagues has been developed meaning that we will emerge a stronger department following COVID with lessons learned that will carry on through to the future.

\section{Compliance with ethical standards}

Conflict of interest Haitham Khashaba, Lisa $\mathrm{Ng}$, Omer Osmani and Richard Chalmers declare no conflict of interest.

Ethical approval No ethical approval required.

Patient consent No patient consent required as no patient data is used.

\section{References}

1. Nicola M, Alsafi Z, Sohrabi C et al (2020) The socio-economic implications of the coronavirus and COVID-19 pandemic: a review. Int J Surg S1743-9191(20):30316-30312

2. Kaye K, Paprottka F, Escudero R, Casabona G, Montes J, Fakin R, Moke L, Stasch T, Richter D, Benito-Ruiz J (2020) Elective, nonurgent procedures and aesthetic surgery in the wake of SARSCOVID-19: considerations regarding safety, Feasibility and Impact on Clinical Management. Aesthet Plast Surg. https://doi. org/10.1007/s00266-020-01752-9

3. Armstrong A, Jeevaratnam J, Murphy G, et al (2020) A plastic surgery service response to COVID-19 in one of the largest teaching hospitals in Europe. J Plast Reconstr Aesthet Surg 73(6):1174 1205. https://doi.org/10.1016/j.bjps.2020.03.027

4. Coronavirus UK map: how many confirmed cases are there in your area? BBC news (2020). https://www.bbc.co.uk/news/uk51768274. Accessed 21 May 2020

5. Peach et al (2020) Available at https://melanomafocus.com/wpcontent/uploads/2020/03/COVID-19-Guide-C-ManagingMelanoma-Patients-during-COVID-19-Pandemic---SurgicalGuidance-for-Clinicians.pdf. Accessed 14 Apr 2020

6. Speciality guide for surgical prioritisation. https://www.england. nhsetups.uk/coronavirus/wp-content/uploads/sites/52/2020/03/ C0221-specialty-guide-surgical-prioritisation-v1.pdf. Accessed 20 May 2020

7. Keith D, De Berker D, Bray A, Brain A, Mustapa MF (2018) British Association of Dermatologists National Audit on nonmelanoma skin cancer excision 2016 in collaboration with the Royal College of Pathologists. BAD newsletter 
8. Lalonde DH (2019) Latest advances in wide awake hand surgery. Hand Clin 35(1):1-6

9. Brown M, Bainbridge C, Wong J, Philips A Wide Awake Hand surgery Handbook https://www.bssh.ac.uk/_userfiles/pages/files/ corona/Wide/Awake/Hand/Surgery/Handbook/v2.pdf. Accessed 30 Mar 2020
10. Fitzgibbon GM (1967) The commandments of Gillies. Br J Plast Surg 21(2-3):226-239. https://doi.org/10.1016/S0007-1226(68) 80028-1

Publisher's note Springer Nature remains neutral with regard to jurisdictional claims in published maps and institutional affiliations. 